\title{
A Train Hot Bearing Detection System Based on Infrared Array Sensor
}

\author{
Wang Ying \\ College of Electronic Information and Control \\ Beijing University of Technology \\ Beijing, China \\ e-mail: wtlwy@bjut.edu.cn
}

\author{
Wu ZhengRong \\ College of Electronic Information and Control \\ Beijing University of Technology \\ Beijing, China \\ e-mail: wzrong1988@emails.bjut.edu.cn
}

\begin{abstract}
Train wheel hot bearing is the primary cause of cutting-axle fracture and severe hotbox accidents, which poses a great threat to the safety of railway transmission. It is necessary and vital to research and develop the technique of hot bearing detector and corresponding system for maintaining the reliable operation of railway system and meeting the requirement of high speed train. In this paper, based on advanced infrared array sensor, a train hot bearing detection system which adopts master-slaver mode, collects bearing temperature array data and uses Bayesian classifier for hotbox discrimination is established to monitor train wheel hot bearing and the detector itself. It provides possibility to improve the accuracy of hotbox alarm, display the waveform of bearing temperature on $3 \mathrm{D}$ and manage the bearing information on whole line.
\end{abstract}

Keywords-infrared array sensor; train hot bearing detection system; Bayesian classifier

\section{INTRODUCTION}

The safe and reliable operation of a railroad system is dependent upon the integrity of the rolling mechanisms of the vehicles traveling over the rails. For example, it is important to monitor a condition of train wheel bearings to determine if a degree of wear on the bearing indicates that the bearings need to be inspected and repaired or replaced. (1) An ignored overheated bearing may collapse and cause train derail, which is extremely disastrous and causes great economic loss. So, it is necessary and vital to research and develop hot bearing detector system for preventing these accidents happened.

The Train Hotbox Detector System currently used in China has made a great contribution for the safety of railway transportation during the past decades. The hot bearing detecting apparatus which it based on made technological innovation continuously and developed from first generation machine to the whole-road networking fourth one. (2) However, there are some general deficiencies in the actual operation of Train Hotbox Detector System such as a high rate of false alarm and a relatively low fulfillment of hotbox, which often causes the train arriving late and then affects the normal order of railway transportation seriously. (3) So with the problems raised above, speeded up of China Railway and rapidly development of computer technology, the more intelligent Hot Bearing Detection system which utilizes information technology and has accurate rate of hot bearing is in demand nowadays.
Generally, the Hot Bearing Detection system uses wayside rail-mounted infrared (IR) transducers to monitor bearing temperature as the train passes by the detector. (4) With the principle of infrared ray, object produces infrared radiation and the radiation energy is proportional to the temperature of the object, the infrared radiation transducer collects bearing temperature of every truck in a non-contact and real-time way. As the foundation of system, the detection technique of wheel temperature sensor largely determinates the serviceability and accuracy of Hot Bearing Detection system. Corresponding to it, the method of identifying hot bearing used in system is important as well.

The paper gives a design of a Train Hot Bearing Detection system based on high-speed wheel temperature digital sensor with infrared array which is different from the detector used generally in China. With relatively rich and reliable bearing temperature data array, it uses Bayesian classifier (5) to identifying the fault level of hot bearing. It has real-time functions of monitoring infrared detector operation and wheel bearing temperature condition, alarming the potential hotbox and storing observation in the database for the whole network sharing. Besides, inquiry of hotbox's details, comparative analysis of distinct hotbox waveform and generation of hot bearing statistics are provided by this system for users.

In Section II the structural design of the Railway Hot Bearing Detection system is mainly discussed; Section IIIdiscuss function applications of the system, data transmission and the discrimination methods of hot bearing in detail; in the final conclusion, some ideas for next work are given.

\section{StRUCtUAL DESIGN OF THE SySTEM}

\section{A. Main Structure of the System}

Considering the independence of construction of the railway hot bearing monitoring network and the security requirements of railway bearing in China, a master-slaver system mode is adopted in order to achieve high-speed acquisition of mass bearing temperature data, accurate analysis and whole-line hot bearing monitoring. The structure of the system is demonstrated in Figure 1.

As figure 1 shown, there are two major parts in this system: detector stations distributed in the trackside and central database server in supervision center. The information of hot bearing and detector collected by different independence detector stations is shared and managed by central database server through Ethernet. 


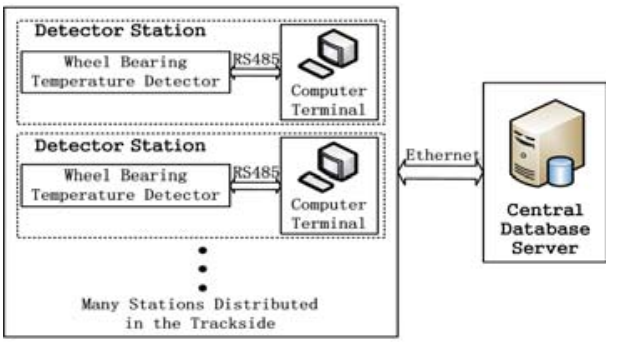

Figure 1. Main Structure of Railway Hot Bearing Detector System

\section{B. The Design of Detector Station}

As the most important component of the Hot Bearing Detection system, every detector station provides central database server with a timely, available and accurate train wheel hotbox information source. Considering the feasibility and accessibility of the system, it consists of two wheel bearing temperature detectors (primary one and auxiliary one), two RS485 transmission lines and a computer terminal as Figure2 illustrated.

- Hot Bearing Detector: It is a high-speed digital detector consisted of an infrared array sensor. When a train passes by the detector, the triggered sensor scans every wheel bearing on 4 parallel points in 32 times at tremendous speed. This means the bearing temperature information of every wheel gathered by the detector is composed of $128(4 \times 32)$ figures. There are two detectors each installed on both side of the track in one detector station. In addition to data acquisition of bearing temperature like the auxiliary one does, the primary one can calculate the serial numbers of truck and bearing, wheelbase, speed and gather the information of detector operation. As shown in Figure2, the above-mentioned figures are sent to computer terminal for analysis and judgment by RS485 interface.

- Computer Terminal: An industrial tablet computer with touch screen is employed to be the hardware of computer terminal which has the characteristics of small size, enough computing power and good performance of anti-seismic, anti-magnetic and antiwet. Base on it, hotbox detection software is developed and uses MYSQL for data management.

- $\quad$ RS485 Transmission Line: High transfer rate, long transfer distance and full-duplex RS485 transmission line whose rate reach $460800 \mathrm{bps}$ is selected as a line for communicating detector with computer terminal. This rate requirement depends on the interval of going through the detector between the passing bearing and the next one. Nowadays, the conventional upper limit of train is $360 \mathrm{Km} / \mathrm{h}$ and the shortest wheelbase is $170 \mathrm{~cm}$. Besides, the data size of bearing information of each pair (right and left) is almost 164MB, data processing and acquisition time is $6 \mathrm{~ms}$ at least. According to above-basis, the rate of transmission line must greater than262400bps.

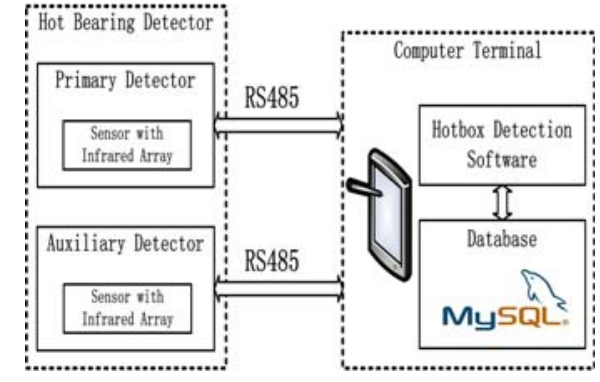

Figure 2. Structure and Composition of Detector Station

\section{Workflow of the System}

When there is no train passes, the detector is on standby. Making use of the hotbox detection software, the terminal can make regular inspection of the detector in this state as Figure3 shown, which means the terminal receives data of detector operation by sending predefined command to it. According to the standards of normal work, the software demonstrates the abnormal condition of detector operation through analyzing obtained data and then lights the alarm lamp.

The detector will be in a state of wheel temperature data acquisition when a coming train is monitored. The temperature data of every wheel bearing collected by infrared array sensors and corresponding truck information will be sent to terminal together in real-time way, then analyze and store in the database as illustrated in Figure3. When the train leaves, the temperature data of every bearing is checked in turn for finding hotbox and discriminating the fault level of hot bearing through the method of hotbox identification in time. If hot bearing exists, the software will alarm users and show the details. Either the result of detector inspection or the details of hot bearing are all stored in the database for sharing, which is helpful for the construction of central database server.

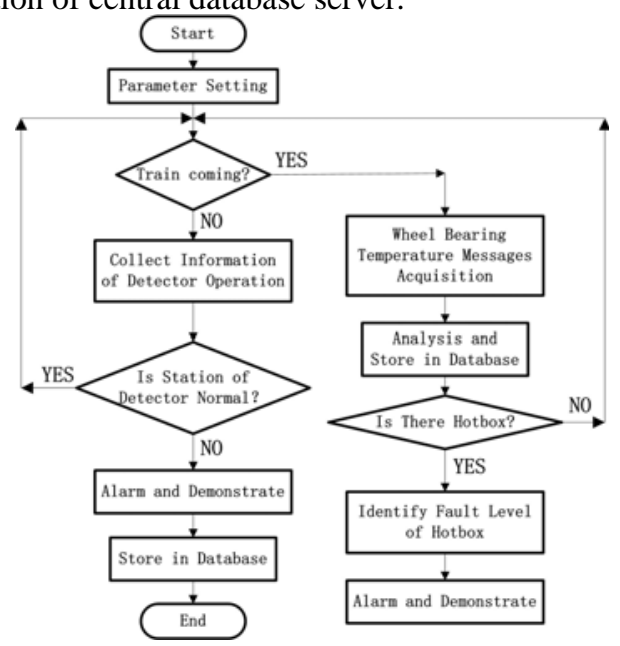

Figure 3. Flow Chart of Hotbox Detection Software 


\section{Hot BEARING DETECTION SyStem APPLICATION}

\section{A. Function of Detector Monitoring}

The good work of the detector is the basis of hot bearing detection. Manual and automatic checking ways are both provided by the system to user. The information of detector condition includes ambient temperature, inside temperature of detector and refrigeration current of main power, the standards of latter parameter change with the former one, which are used to estimating whether or not the detector is in normal operation. Figure4 shows a test result of infrared array detector monitoring in lab.

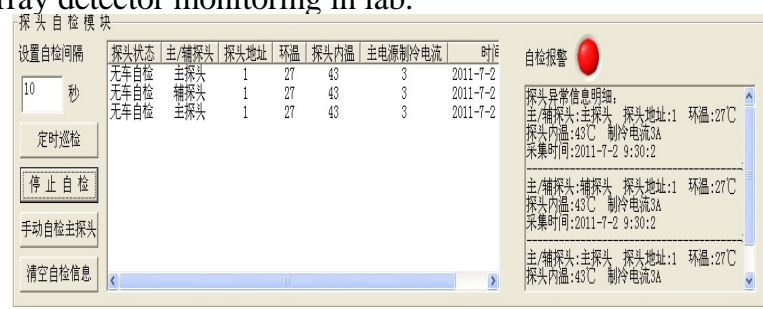

Figure 4. A Test Result of Detector Monitoring in Lab

\section{B. Discrimination of Hot Bearing}

Due to poor lubrication, the wheel bearing will generate more heat than normal operation after failure. Reflecting in bearing temperature, it is several times the normal work, that's how hot bearing forms. Generally, it is divided into three levels for alarming: micro heat, intense heat and shocked heat. They are manually set and describe the generate possibility of hotbox due to high wheel bearing temperature.

Temperature-rising is a direct factor to determine hot bearing, so the basic method for distinguishing hotbox is comparing temperature-rising of the wheel bearing with the standard. It is obviously undesirable and imprecise to determine the fault degree of hotbox by relying on only one factor. So there are three parameters are adopted by the system for discrimination: temperature-rising, train difference of temperature-rising and truck difference of temperature-rising.

As introduced in Section II , bearing temperature data collected by the infrared array detector is four times as big as original technique. The schematic diagram of temperature waveform of a bearing is shown in Figure5.

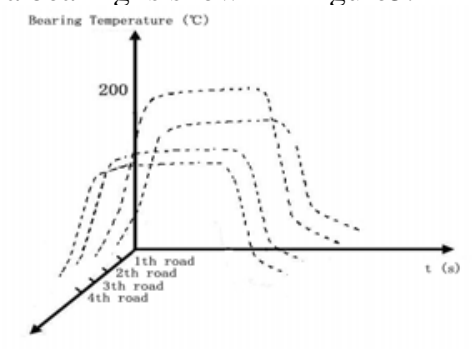

Figure 5. Temperature Waveform of a Bearing
According to the condition of detector in the system, the three parameters are represented respectively by Equation (1),(2),(3) as below.

$$
\left\{\begin{array}{l}
\Delta T_{i}=T_{i}-T_{0} \\
T_{i}=\frac{\max _{32} T_{i 1}+\max _{32} T_{i 2}+\max _{32} T_{i 3}+\max _{32} T_{i 4}}{4}
\end{array} .\right.
$$

\section{$\Delta T_{i}$ : Temperature-rising of bearing;}

$T_{i}$ : A bearing temperature;

$T_{0}$ : Ambient temperature;

$\max _{32} T_{i 1}, \max _{32} T_{i 2}, \max _{32} T_{i 3}, \max _{32} T_{i 4}:$ The maximum of first, second, third and forth road bearing temperature;

$$
\Delta \mathrm{X}_{\mathrm{i}}=\mathrm{T}_{i}-\frac{\sum_{j=1}^{N} T_{j}}{N} .
$$

$\Delta \mathrm{X}_{\mathrm{i}}$ : Train difference of temperature-rising;

$T_{j}$ : A bearing temperature;

$\mathrm{N}$ : The bearing number of the whole train which includes the bearing and in the same side with it.

$$
\Delta \mathrm{Y}_{\mathrm{i}}=\mathrm{T}_{i}-\frac{\sum_{j=1}^{M} T_{j}}{M} \text {. }
$$

$\Delta \mathrm{Y}_{\mathrm{i}}$ : Truck difference of temperature-rising;

$T_{j}$ : A bearing temperature;

M: The bearing number of the whole truck which includes the bearing and in the same side with it.

Instead of judging the value of $\Delta T_{i}, \Delta X_{i}$ and $\Delta Y_{i}$ falls in the fixed standard interval whether or not, mature naive Bayesian classifier (5) is used in the system for hotbox discrimination. As the characteristic properties of hot bearing, the value of $\Delta T_{i}, \Delta X_{i}$ and $\Delta Y_{i}$ are affected by some factors, such as ambient temperature, type of bearing, condition of detector operation, installation site of the detector and so on. So depending on the different situation, they are divided into four ranges of heat: normal, micro, intense and shocked. Then the fault level of hotbox is graded through the corresponding classifier generated by training samples: mass bearing temperature data which has been identified artificially. This method of hotbox discrimination makes great advantage of rich temperature data gathered by infrared array sensor.

\section{Function of Wheel Bearing Information Query and Statistic}

The system provides users with querying detected bearing information based on time and train number condition. The query result displays in a list, moreover, the waveforms of selected hot bearings are demonstrated on 2D or 3D for analyzing and comparing by using Measurement Studio controller as Figure5 shown. It should be mentioned that the data illustrated in Figure6 is simulate temperature data for query function test.

The statistical function is used for offering users some useful and macro information of detected wheel bearing 
during a selected period of time, such as the total number of detected trains and bearings and the situation of hot bearing distribution.

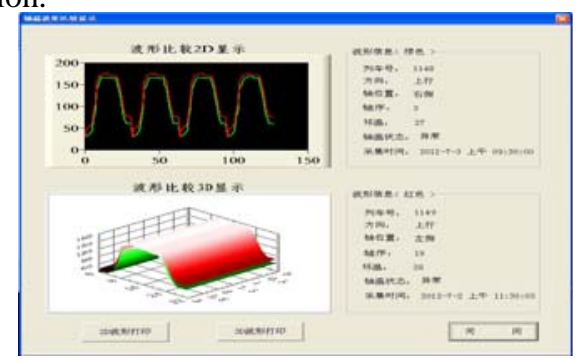

Figure 6. A Contrast of Two Selected Hot Bearing

\section{CONCLUSION}

A train Hot Bearing Detection system based on wheel temperature infrared array sensor is given in this paper for alarming wheel hotbox timely and protecting the safety of railway transportation. Rich bearing temperature data collected by the infrared array sensor and the utilization of Bayesian classifier (5) make it possible to improve the accuracy of the hotbox alarm. The system also has advantages in displaying waveforms on $3 \mathrm{D}$ and managing the whole bearing information in a master-slaver mode, which are helpful for analyzing and comparing bearing temperature.
Aiming at holding the distribution situation of hot bearing in the whole line and providing a platform for comprehensive analysis to users, the web application based on the central database server is still in the development. However, train hot bearing detection system is the main part and it would be improved and perfected by the more practice and further study.

\section{REFERENCES}

[1] Peter Hesser, Thomas Strecker, Miles Metschke, Daniel Kurt Stevens, Mathias Hartmann. Train Wheel Bearing Temperature Detector: United States, US2006/0131464 A1[P], Jun.22,2006.

[2] Zhao ChangBo, Chen Lei. "The Introduction of Railway Truck Hotbox Detection and Application”, China Railway Publishing House, Bei Jing, 2010.

[3] Liu ZhanGong. "The Analysis and Recommendations of False Hotbox Alarm in Infrared Bearing Detection System”, Railway Locomotive \& Car, Vol.26 No.4, 2006.

[4] Howard C. Choe, Yulun Wan, Andrew KChan. "Neural Pattern Identification of Railroad Wheel-bearing Faults from Audible Acoustic Signals: Comparison of FFT, CWT, and DWT Features”, Proceedings of the SPIE (3078), 1997.

[5] Arpad Kelemen, Hong Zhou, Pamela Lawhead, Yulan Liang. "Naive Bayesian classifier for microarray data", Neural Networks, 2003. Proceedings of the International Joint Conference on, 1769-1773 vol.3. 